\title{
A NOTE ON A PROBLEM IN ASYMPTOTICS
}

\author{
BY
}

R. SHAIL

Department of Mathematics and Statistics, University of Surrey, Guildford, Surrey GU2 5XH, UK

Abstract. This note examines the asymptotic evaluation of two infinite series considered recently in [1] and [2]. After a transformation to a more convenient form, elementary methods are used to derive extended asymptotic results (in one case the complete asymptotic expansion in the appropriate limit), and the numerical performance of the expansions is illustrated.

1. Introduction. In [1] Dhondt and Köhl have solved the two-dimensional problem of a dielectric cylinder placed in front of a conducting half-space, there being a constant electric field applied perpendicular to the half-space. Using a bipolar coordinate system, the potential field is determined, and the force on the cylinder is shown to be proportional to $F_{d}$, where

$$
\begin{gathered}
F_{d}=\left(1-\kappa^{2}\right) \sinh u_{0} \sum_{n=1}^{\infty}\left\{\frac{n(n+1) \cosh u_{0}}{\left(\kappa \sinh n u_{0}+\cosh n u_{0}\right)\left(\kappa \sinh (n+1) u_{0}+\cosh (n+1) u_{0}\right)}\right. \\
\left.-\frac{n^{2}}{\left(\kappa \sinh n u_{0}+\cosh n u_{0}\right)^{2}}\right\} \\
=\left(1-\kappa^{2}\right)\left(S_{1}-S_{2}\right)
\end{gathered}
$$

in an obvious notation. In (1), the radius of the cylinder is taken to be unity and $c=\cosh u_{0}$ is the distance of the cylinder axis from the conducting half-space; further, $\kappa=\epsilon / \epsilon_{0}$ is the ratio of the cylinder dielectric permittivity to that of free space. The value $F_{c}$ for a conducting cylinder is obtained from (1) in the limit $\kappa \rightarrow \infty$ as

$$
F_{c}=-\sinh u_{0} \sum_{n=1}^{\infty}\left\{\frac{n(n+1) \cosh u_{0}}{\sinh n u_{0} \sinh (n+1) u_{0}}-\frac{n^{2}}{\sinh ^{2} n u_{0}}\right\} .
$$

The authors of [1] then derive the leading terms in the asymptotic developments of (1) and (3) as $u_{0} \rightarrow 0$, i.e., the cylinder approaches the plane face of the conducting half-space. The mathematical arguments used are somewhat long and intricate, and a

Received October 14, 1998.

2000 Mathematics Subject Classification. Primary 34E05. 
crucial step (the replacement of $\sinh n u_{0} \sinh (n+1) u_{0}$ in $(3)$ by $\sinh ^{2} n u_{0}$ ) is justified at length in [2]. Specifically, it is found that

$$
F_{d} \sim \frac{\lambda^{2}}{3}\left(\frac{1}{1+\lambda}-Q(\lambda)\right) \quad \text { and } \quad F_{c} \sim \frac{1}{2 u_{0}}
$$

as $u_{0} \rightarrow 0$, where

$$
\lambda=\frac{1-\kappa}{1+\kappa} \quad \text { and } \quad Q(\lambda)=\sum_{j=0}^{\infty} \frac{(-\lambda)^{j}}{(j+2)^{2}} .
$$

The object of this note is to compute in a simple manner higher-order asymptotic expansions of (1) and (3). Although (1) and (3) can be expanded asymptotically as they stand, it turns out that a preliminary transformation of the infinite series produces much simpler and preferable representations of $F_{d}$ and $F_{c}$. This transformation is described in Sec. 2 (see (11) and (12)). The appropriate asymptotic expansion for $-1<\lambda<1$ of $F_{d}$ as $u_{0} \rightarrow 0$ is then obtained, correct to $O\left(u_{0}^{8}\right)$, by expanding the summand in (11) in powers of $u_{0}$ to the desired order, followed by summation over $n$. The numerical performance of the expansion is examined and its limitations are noted.

When $\lambda=-1$ the sums over $n$ referred to in the previous paragraph become divergent. However, following Rawlins [3], the complete Poincaré asymptotic expansion of $F_{c}$ in (13) as $u_{0} \rightarrow 0$ can be found by an application of the Euler-Maclaurin sum formula to a suitably defined function. This calculation is carried out in Sec. 3 and the numerical accuracy of the expansion is assessed. Alternative expansions are also developed for $F_{c}$ in terms of $c$, the distance of the cylinder axis from the face of the conducting half-space. The note concludes with a further comment on the work in [1] and [2].

2. The asymptotic expansion of $F_{d}$ as $u_{0} \rightarrow 0$. We first carry out the transformation of (1) alluded to earlier. Consider the infinite series $S_{1}$ defined by (1) and (2); converting the hyperbolic functions to exponentials, this can be rewritten in terms of $\lambda$ (defined in $\left.(5)_{1}\right)$ as

$$
\begin{aligned}
S_{1} & =\frac{4 \sinh u_{0} \cosh u_{0}}{(\kappa+1)^{2}} \sum_{n=1}^{\infty} \frac{n(n+1) e^{-(2 n+1) u_{0}}}{\left(1+\lambda e^{-2 n u_{0}}\right)\left(1+\lambda e^{-2(n+1) u_{0}}\right)} \\
& =-\frac{2 \cosh u_{0}}{\lambda(\kappa+1)^{2}} \sum_{n=1}^{\infty} n(n+1)\left\{\frac{1}{1+\lambda e^{-2 n u_{0}}}-\frac{1}{1+\lambda e^{-2(n+1) u_{0}}}\right\} .
\end{aligned}
$$

Since $-1 \leq \lambda \leq 1$ and $u_{0}>0$, we can expand $\frac{1}{1+\lambda e^{-2 n u_{0}}}$ and $\frac{1}{1+\lambda e^{-2(n+1) u_{0}}}$ binomially to give

$$
S_{1}=-\frac{2 \cosh u_{0}}{\lambda(\kappa+1)^{2}} \sum_{n=1}^{\infty} n(n+1)\left\{\sum_{m=1}^{\infty}(-\lambda)^{m}\left(e^{-2 n m u_{0}}-e^{-2(n+1) m u_{0}}\right)\right\} .
$$

The orders of summation can now be interchanged, and the inner summation effected using the result that

$$
\sum_{n=1}^{\infty} n(n+1)\left(e^{-n y}-e^{-(n+1) y}\right)=\frac{1}{2 \sinh ^{2}(y / 2)}
$$


for $y>0$. We obtain

$$
S_{1}=-\frac{\cosh u_{0}}{\lambda(\kappa+1)^{2}} \sum_{n=1}^{\infty} \frac{(-\lambda)^{n}}{\sinh ^{2} n u_{0}} .
$$

In a similar manner it is found that

$$
S_{2}=-\frac{\sinh u_{0}}{\lambda(\kappa+1)^{2}} \sum_{n=1}^{\infty} \frac{n(-\lambda)^{n} \cosh n u_{0}}{\sinh ^{3} n u_{0}} .
$$

Substituting (9) and (10) into (2) now shows, on simplification, that

$$
F_{d}=\sum_{n=1}^{\infty}(-\lambda)^{n+1} \frac{\left(n \sinh u_{0} \cosh (n+1) u_{0}-\sinh n u_{0}\right)}{\sinh ^{3}(n+1) u_{0}} .
$$

Setting $\lambda=-1$ gives for the conducting-cylinder case that

$$
F_{c}=\sum_{n=1}^{\infty} \frac{\left(n \sinh u_{0} \cosh (n+1) u_{0}-\sinh n u_{0}\right)}{\sinh ^{3}(n+1) u_{0}}
$$

With some slight manipulation, (12) can be written as

$$
F_{c}=-\cosh u_{0} \sum_{n=1}^{\infty} \frac{1}{\sinh ^{2} n u_{0}}-\frac{\sinh u_{0}}{2} \frac{d}{d u_{0}} \sum_{n=1}^{\infty} \frac{1}{\sinh ^{2} n u_{0}} .
$$

The form (11) for $F_{d}$ is clearly simpler and superior to (1); the dependence on the material properties of the dielectric appears only in the power $(-\lambda)^{n+1}$, rather than in denominator factors as in (1). Further, it contains no products of the form $\sinh n u_{0} \sinh (n+1) u_{0}$ which caused such complications in the asymptotic analysis of (3) in [1] and [2].

We now turn to the asymptotic expansion as $u_{0} \rightarrow 0$ of (11) and suppose that $|\lambda|<1$. The summand is first expanded about $u_{0}=0$ to the required asymptotic order; thus, for example, to $O\left(u_{0}^{2}\right)$ we have that

$$
F_{d}=\sum_{n=1}^{\infty}(-\lambda)^{n+1}\left\{\frac{n(n+2)}{3(n+1)^{2}}-\frac{n\left(4 n^{3}+16 n^{2}+19 n+6\right)}{30(n+1)^{2}} u_{0}^{2}\right\}+O\left(u_{0}^{4}\right) .
$$

Using partial fractions,

$$
\begin{aligned}
\sum_{n=1}^{\infty}(-\lambda)^{n+1} \frac{n(n+2)}{(n+1)^{2}} & =\sum_{n=1}^{\infty}(-\lambda)^{n+1}-\sum_{n=1}^{\infty} \frac{(-\lambda)^{n+1}}{(n+1)^{2}} \\
& =\lambda^{2}\left(\frac{1}{1+\lambda}-Q(\lambda)\right)
\end{aligned}
$$

for $|\lambda|<1$. The result $(4)_{1}$ now follows immediately and terms of higher order in $u_{0}$ can be similarly calculated.

This process can be implemented using the standard functions of any computer algebra system and the expansion of $F_{d}$ generated to the required order in $u_{0}$. Thus, with an 
error of order $u_{0}^{10}$, it is found that

$$
\begin{aligned}
& F_{d}=\frac{\lambda^{2}}{3}\left(\frac{1}{1+\lambda}-Q(\lambda)\right)+\frac{\lambda^{2}}{30}\left(\frac{\lambda^{2}-2 \lambda-11}{(1+\lambda)^{3}}-Q(\lambda)\right) u_{0}^{2} \\
& +\frac{\lambda^{2}}{2520}\left(\frac{3 \lambda^{4}-20 \lambda^{3}-142 \lambda^{2}-1172 \lambda+867}{(1+\lambda)^{6}}-3 Q(\lambda)\right) u_{0}^{4} \\
& +\frac{\lambda^{2}}{45360}\left(\frac{\lambda^{6}-14 \lambda^{5}-125 \lambda^{4}+10988 \lambda^{3}-85865 \lambda^{2}+85186 \lambda-11387}{(1+\lambda)^{7}}-Q(\lambda)\right) u_{0}^{6} \\
& +\frac{\lambda^{2}}{3991680}\left(\frac{P(\lambda)}{(1+\lambda)^{9}}-Q(\lambda)\right) u_{0}^{8}+O\left(u_{0}^{10}\right),
\end{aligned}
$$

where

$$
\begin{aligned}
P(\lambda)= & \lambda^{8}-24 \lambda^{7}-260 \lambda^{6}-643432 \lambda^{5}+15295398 \lambda^{4} \\
& -61476904 \lambda^{3}+61472956 \lambda^{2}-15298008 \lambda+642337 .
\end{aligned}
$$

It is appropriate to make some comments on (16) at this point. We first note that the elementary process used cannot be applied to (1). The first terms of the expansion about $u_{0}=0$ of the summand in $S_{1}-S_{2}$ of (2) are

$$
n u_{0}-n(3 n+1) \kappa u_{0}^{2}+O\left(u_{0}^{3}\right)
$$

and subsequent summations over $n$ are divergent. An approach such as the method of inner and outer sums (see [4], [5]) can be used but the calculation is much more complicated. Although derived on the assumption that $|\lambda|<1$, (16) remains valid for $\lambda=1$; this can be verified $a b$ initio by applying the method of inner and outer sums as developed in [5] for alternating series.

For positive $\lambda$, the approximation (16) is accurate for values of $u_{0}$ up to 0.7. This is illustrated for $\lambda=0.9$ in Fig. 1 which shows the graphs of the $O\left(u_{0}^{8}\right)$-approximation (dotted line) and the infinite series (11) with the upper limit replaced by 100 (solid line).

However, it is clear that the presence of inverse powers of $1+\lambda$ in (16) will restrict its usefulness to progressively smaller ranges of $u_{0}$ as $\lambda \rightarrow-1+$. Thus, when $\lambda=-0.9$, Fig. 2 shows that (16) (dotted line) is only accurate for $\lambda$ between 0 and about 0.03 (again the solid line is the graph of (11)). The determination of an asymptotic approximation valid for a larger range of $u_{0}$ when $\lambda$ approaches -1 remains an open problem.

We conclude this section by noting an approximation to (16) that is valid when the cylinder is a large distance from the half-space, i.e., $c \gg 1$. Since $c=\cosh u_{0}$, it follows that $\exp u_{0}=c+\sqrt{c^{2}-1}$. Thus we can express the hyperbolic functions in (16) in terms of $c$ and use symbolic manipulation to find the asymptotic form of the various terms in 


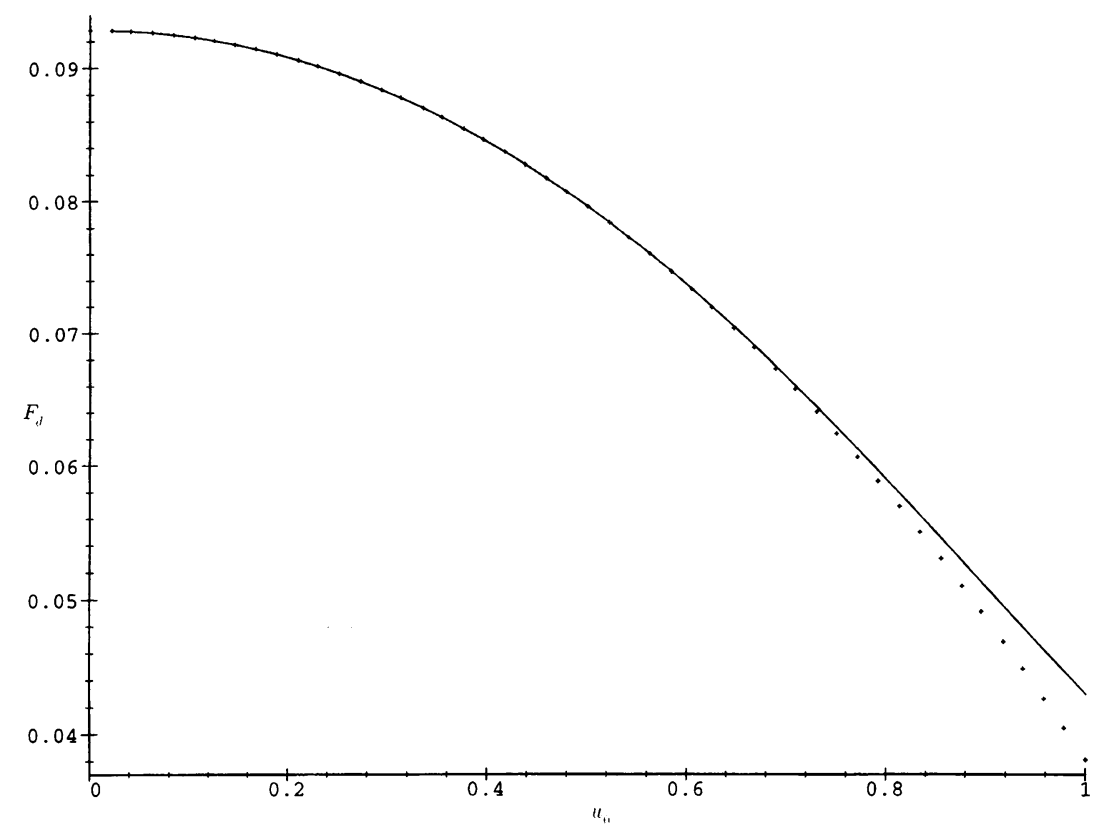

FIG. 1. Graphs of $F_{d}$ for $\lambda=0.9$ given by (16) (dotted line) and series (11) (solid line).

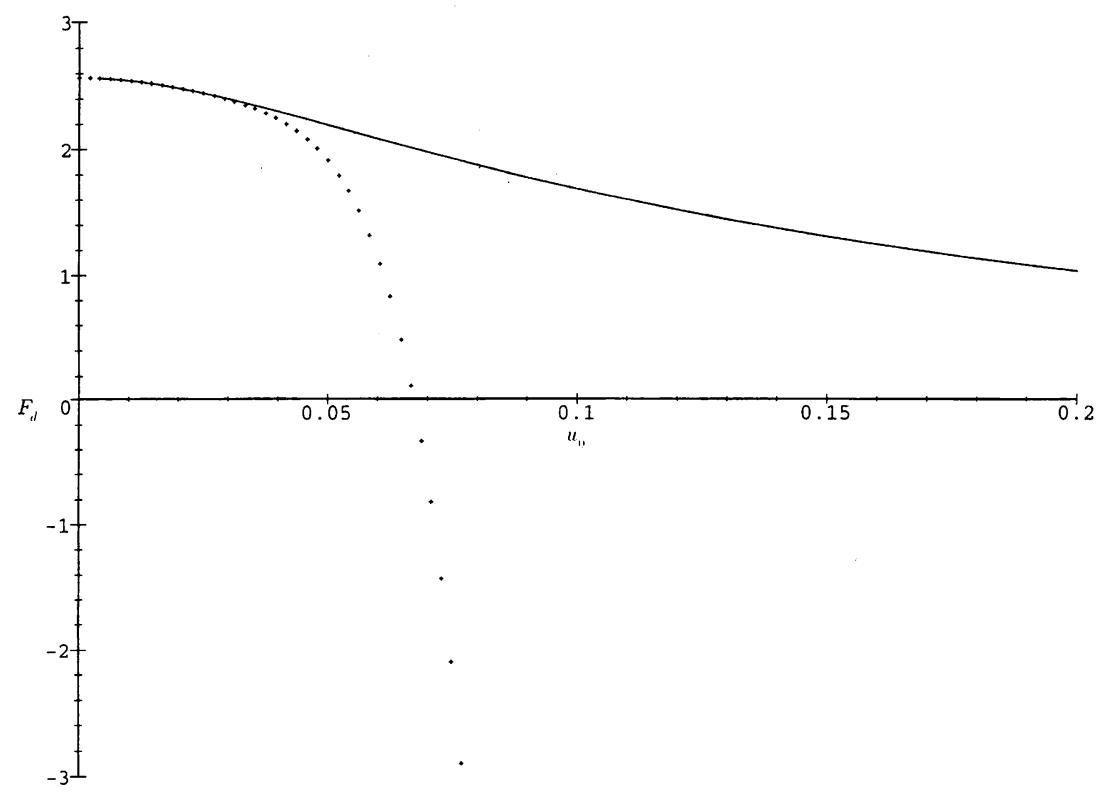

FIG. 2. Graphs of $F_{d}$ for $\lambda=-0.9$ given by (16) (dotted line) and series (11) (solid line). 
the sum as $c \rightarrow \infty$. Correct to $O\left(1 / c^{17}\right)$,

$$
\begin{aligned}
F_{d}= & \frac{1}{4} \frac{\lambda^{2}}{c^{3}}-\frac{1}{8} \frac{\lambda^{3}}{c^{5}}+\frac{3}{64} \frac{\lambda^{3}(\lambda-2)}{c^{7}}-\frac{1}{64} \frac{\lambda^{3}(\lambda-1)(\lambda-3)}{c^{9}} \\
& +\frac{5}{1024} \frac{\lambda^{3}\left(12 \lambda+\lambda^{3}-4-6 \lambda^{2}\right)}{c^{11}}-\frac{3}{2048} \frac{\lambda^{3}\left(25 \lambda^{2}-8 \lambda^{3}+\lambda^{4}-32 \lambda+5\right)}{c^{13}} \\
& +\frac{7}{16384} \frac{\lambda^{3}\left(-10 \lambda^{4}-90 \lambda^{2}+80 \lambda+42 \lambda^{3}+\lambda^{5}-6\right)}{c^{15}} \\
& -\frac{1}{8192} \frac{\lambda^{3}\left(7+\lambda^{6}-192 \lambda-184 \lambda^{3}+63 \lambda^{4}-12 \lambda^{5}+300 \lambda^{2}\right)}{c^{17}}+O\left(\frac{1}{c^{19}}\right) .
\end{aligned}
$$

3. The asymptotic expansion of $F_{c}$ as $u_{0} \rightarrow 0$. It is clear from the expression given for $F_{c}$ in (13) that we require the asymptotic expansion as $u_{0} \rightarrow 0$ of

$$
\sum_{n=1}^{\infty} \frac{1}{\sinh ^{2} n u_{0}}
$$

We follow the approach of Rawlins [3] and define a function $f(x)$ for all $x$ by

$$
f(x)=\left\{\begin{array}{cc}
\frac{1}{\sinh ^{2} u_{0} x}-\frac{1}{u_{0}^{2} x^{2}}, & x \neq 0 \\
-\frac{1}{3}, & x=0 .
\end{array}\right.
$$

Then $f(x)$ is an even continuous function of $x$ that tends to zero as $x \rightarrow \infty$; further, all its odd-order derivatives vanish as $x \rightarrow 0$ and $x \rightarrow \infty$. With these observations, the Euler-Maclaurin sum formula reduces to

$$
\sum_{n=1}^{\infty} f(n) \sim \int_{0}^{\infty} f(x) d x-\frac{1}{2} f(0)
$$

Using the definition (20) and the results

$$
\int_{0}^{\infty}\left(\frac{1}{\sinh ^{2} u_{0} x}-\frac{1}{u_{0}^{2} x^{2}}\right) d x=-\frac{1}{u_{0}} \quad \text { and } \quad \sum_{n=1}^{\infty} \frac{1}{n^{2}}=\frac{\pi^{2}}{6}
$$

in $(21)$, it now follows that

$$
\sum_{n=1}^{\infty} \frac{1}{\sinh ^{2} n u_{0}} \sim \frac{\pi^{2}}{6 u_{0}^{2}}-\frac{1}{u_{0}}+\frac{1}{6}
$$

The error in (22) is $O\left(u_{0}^{k}\right)$ as $u_{0} \rightarrow 0$ for any integer $k \geq 1$, and combining (22) with (13) we have that

$$
F_{c} \sim-\cosh u_{0}\left(\frac{\pi^{2}}{6 u_{0}^{2}}-\frac{1}{u_{0}}+\frac{1}{6}\right)-\frac{\sinh u_{0}}{2}\left(-\frac{\pi^{2}}{3 u_{0}^{3}}+\frac{1}{u_{0}^{2}}\right),
$$




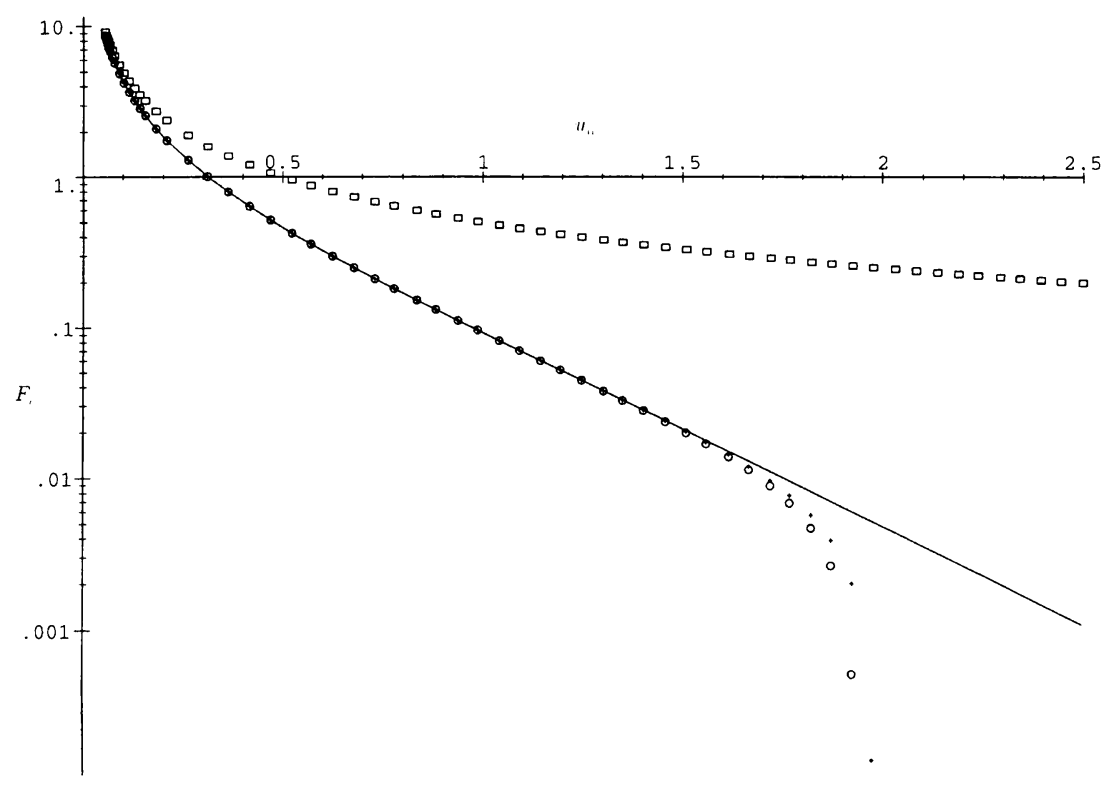

FIG. 3. Log plots of $F_{c}$ given by (23) (dotted line), (24) (o), 1/(2u $)$

$(\square)$ and series (12) (solid line).

the complete asymptotic expansion of $F_{c} \cdot{ }^{1}$ The right-hand side of (23) can be expanded in powers of $u_{0}$ to any desired order; thus, for example,

$$
\begin{aligned}
F_{c}= & \frac{1}{2 u_{0}}-\frac{1}{6}-\frac{\pi^{2}}{18}+\frac{5}{12} u_{0}-\left(\frac{1}{12}+\frac{\pi^{2}}{180}\right) u_{0}^{2}+\frac{3}{80} u_{0}^{3} \\
& -\left(\frac{1}{144}+\frac{\pi^{2}}{5040}\right) u_{0}^{4}+\frac{13}{10080} u_{0}^{5}-\left(\frac{1}{4320}+\frac{\pi^{2}}{272160}\right) u_{0}^{6}+O\left(u_{0}^{7}\right)
\end{aligned}
$$

as $u_{0} \rightarrow 0$. The leading term of (24) agrees with that found in [1]. The remarkable accuracy of (23) and (24) for $0<u_{0}<1.5$ is illustrated in Fig. 3; in this range the log plots against $u_{0}$ of (23) (dotted line), (24) (o) and the sum (12) with an upper limit of 100 (solid line) are virtually indistinguishable. For comparison purposes, the log plot of the leading term in (24) ( $\square$ ) is also shown.

In the case $c \gg 1$, the appropriate asymptotic development of $F_{c}$ can be found as described in Sec. 2. Taken to a somewhat extravagant order of approximation, it is found that

$$
\begin{aligned}
F_{c}=\frac{1}{4 c^{3}} & +\frac{1}{8 c^{5}}+\frac{9}{64 c^{7}}+\frac{1}{8 c^{9}}+\frac{115}{1024 c^{11}}+\frac{213}{2048 c^{13}}+\frac{1603}{16384 c^{15}}+\frac{759}{8192 c^{17}} \\
& +\frac{11547}{131072 c^{19}}+\cdots+\frac{987443849289}{17592186044416 c^{49}}+O\left(\frac{1}{c^{51}}\right) .
\end{aligned}
$$

The terms to $O\left(1 / c^{17}\right)$ in (25) agree with those obtained by setting $\lambda=-1$ in (18).

\footnotetext{
${ }^{1}$ For readers alarmed by the dire warnings given in textbooks against differentiating asymptotic expansions, the expansion of $\sum_{n=1}^{\infty} n \cosh n u_{0} / \sinh ^{3} n u_{0}$ can be verified using the Euler-Maclaurin sum formula as for (22).
} 
It follows from (24) that as $c \rightarrow 1, F_{c}$ becomes singular like $1 / \sqrt{c-1}$. Extracting this singularity from (25) in the manner described by Van Dyke [6], an alternative approximation to $F_{c}$ is

$$
\begin{aligned}
F_{c}= & \sqrt{\frac{c}{c-1}}\left(\frac{1}{4 c^{3}}-\frac{1}{8 c^{5}}+\frac{3}{32 c^{5}}-\frac{5}{64 c^{6}}+\frac{59}{512 c^{7}}-\frac{87}{1024 c^{8}}\right. \\
& \left.+\frac{399}{4096 c^{9}}+\cdots+\frac{49981285358230685953246257}{1237940039285380274899124224 c^{49}}+O\left(\frac{1}{c^{50}}\right)\right) .
\end{aligned}
$$

Table 1 contains some representative numerical values of $F_{c}$ for various $c$ calculated from (23), (25), and (26).

Table 1

$\begin{array}{llll}c & F_{c},(23) & F_{c},(26) & F_{c},(25) \\ 2.0 & 0.03650 & 0.03657 & 0.03657 \\ 1.1 & 0.57283 & 0.57327 & 0.57055 \\ 1.01 & 2.87972 & 2.87954 & 1.75966 \\ 1.001 & 10.48465 & 9.89506 & 2.10373 \\ 1.0001 & 34.64652 & 31.57700 & 2.14365\end{array}$

The accuracy of $(26)$ is impressive; for $c=1.01$, that is, the cylinder is $1 / 100$ of a radius from the half-space, a formula derived from the large-separation result is in error by less than $0.02 \%$ !

We conclude this note by returning to the analysis in [1] and [2], where a crucial step is the proof that the replacement of the denominator $\sinh n u_{0} \sinh (n+1) u_{0}$ in (3) by $\sinh ^{2} n u_{0}$ does not alter the leading term in the asymptotic expansion as $u_{0} \rightarrow 0$. Using the method of inner and outer sums we can show that

$$
\begin{aligned}
-\sinh u_{0} \sum_{n=1}^{\infty} \frac{n(n+1) \cosh u_{0} \sinh n u_{0}-n^{2} \sinh (n+1) u_{0}}{\sinh ^{3} n u_{0}} \\
\sim \frac{1}{2 u_{0}}-\left(\frac{7}{36}-\frac{1}{3} \Delta\right) u_{0}-\left(\frac{233}{2700}+\frac{4}{25} \Delta\right) u_{0}^{3} \\
-\left(\frac{9781}{793800}+\frac{1}{105} \Delta\right) u_{0}^{5}+O\left(u_{0}^{7} \ln u_{0}\right)
\end{aligned}
$$

where $\Delta=\gamma-\ln 2 u_{0}$ and $\gamma$ is Euler's constant. Comparing (24) and (27) we see explicitly that the leading terms agree; however, subsequent terms have a very different structure and $u_{0}$-dependence. Thus it would seem that the methods used in [1] and [2] cannot be extended to higher order in $u_{0}$. It is also worth noting that, as proved in [2], the nonuniform convergence for $u_{0} \in[0, \delta], \delta>0$, of (13) can be established quickly using the inequality in Theorem 3 of [2] and the elementary result that

$$
\frac{1}{\sinh ^{2} n u_{0}}>\frac{4}{e^{2 n u_{0}}}
$$

for $u_{0}>0$. 


\section{REFERENCES}

[1] G. Dhondt and M. Köhl, On the force between a dielectric cylinder in a constant electric field and a conducting half space, Quart. Appl. Math. 55, 347-359 (1997)

[2] G. Dhondt, On the summation of a particular non-uniformly-convergent series, Quart. Appl. Math. 56, 107-114 (1998)

[3] A. D. Rawlins, Note on the capacitance of two closely separated spheres, IMA J. Applied Math. 34, 119-120 (1985)

[4] R. G. Cox and H. Brenner, The slow motion of a sphere through a viscous fluid towards a plane surface -II. Small gap widths including inertial effects, Chem. Engrg. Sci. 22, 1753-1777 (1967)

[5] R. Shail, On the asymptotic expansion of certain functions defined by infinite series, Mathematika 44, 401-418 (1997)

[6] M. Van Dyke, Analysis and improvement of perturbation series, Quart. J. Mech. Appl. Math. 27, 423-450 (1974) 